\title{
A conceptual design of fouling deposit adhesiveness gauge
}

\begin{abstract}
Fouling deposit is an unwanted layer that forms in most of the processing equipment. It affects the product quality, economics and plant efficiency. Information on interfacial forces between fouling deposit and equipment surface is essential for finding optimum cleaning. The objective of this work is to provide a conceptual design of a prototype for a gauge that can measure the adhesiveness of fouling deposit to the stainless steel surface. In this work drag force concept is applied and it is assumed proportional to the magnitude of the adhesion strength of the fouling deposit to the stainless surface. This design will allow non-invasive measurement.
\end{abstract}

Keyword: Drag force; No-invasive method 